\title{
Fachbereich Wirtschaftswissenschaften
}

\author{
Universität Konstanz
}

\section{Ist eine duale Einkommensteuer einfacher und gerechter als eine umfassende Einkommensteuer?}

Bernd Genser (Universität Konstanz)

Oktober 2001

Schriftliche Ausarbeitung eines Vortrages am Steuerkongress des Heidelberger Kreises, 21.22. Juni 2001, Universität Heidelberg.

Korrespondenzadresse:

Prof. Dr. Bernd Genser

Universität Konstanz

Fach D133

Telefon: +49 (07531) 88-2345

D-78457 Konstanz,

Deutschland

Fax: $\quad+49$ (07531) 88-4101

E-mail: bernd.genser@uni-konstanz.de

Internet: http://finance.wiwi.uni-konstanz.de 


\title{
Ist eine duale Einkommensteuer einfacher und gerechter als eine umfassende Einkommensteuer?
}

\author{
Bernd Genser (Universität Konstanz)
}

\section{$1 \quad$ Einleitung ${ }^{1}$}

Die umfassende Einkommensteuer ist ein Leitbild der Steuerpolitik, das aus ökonomischer Sicht den finanzwissenschaftlichen Grundsätzen eines rationalen und gerechten Steuersystems entspricht und sich im demokratischen Willensbildungsprozess in praktisch allen entwickelten Volkswirtschaften als gesellschaftlich mehrheitsfähig erwiesen hat.

Das Konzept der umfassenden Einkommensteuer, das nach seinen historischen Befürwortern Georg von Schanz, Murray Haig und Henry Simons auch als Schanz/Haig/Simons-Standard (kurz SHS-Konzept) der Einkommensbesteuerung bezeichnet wird, legt zwei Fundamentalprinzipien für die Einkommensteuerbasis fest.

Erstens definiert das Syntheseprinzip als steuerpflichtiges Einkommen die Summe aller (positiven und negativen) Einkünfte, die dem Steuerpflichtigen während eines Steuerjahres zufließen. Dadurch unterscheidet sich die synthetische Einkommensteuer von einer analytischen Einkommensteuer (Schedulensteuer), die je nach Einkunftsart unterschiedliche Steuerbasen festlegt, die dann auch unterschiedlich besteuert werden können.

Zweitens definiert das Reinvermögenszugangsprinzip als steuerpflichtiges Einkommen die Wertänderung aller Sach- und Finanzvermögensanlagen eines Steuerpflichtigen während eines Steuerjahres, einschließlich jener Neuveranlagung von Vermögenswerten, die dann möglich gewesen wäre, wenn der Steuerpflichtige auf alle seine Konsumaktivitäten verzichtet und deren monetären Gegenwert ebenfalls zur Kapitalakkumulation verwendet hätte. Nicht als potentielle Vermögenszuwächse gewertet werden hingegen alle jene Aufwendungen, die dem Steuerpflichtigen im Zusammenhang mit dem Erwerb von Einkünften erwachsen (Nettoprinzip). Die umfassende Einkommensteuer unterscheidet sich damit von einer Besteuerung nach dem Quellenprinzip, bei dem nur die aus Vermögenswerten regelmäßig zufließenden Erträge steuerpflichtig sind, oder von einer persönlichen Ausgabensteuer, bei der nur jener Teil des umfassenden Einkommens steuerpflichtig ist, der konsumtiv verwendet wird.

Die deutsche Einkommensbesteuerung folgt der Leitidee der umfassenden Einkommensteuer. Dies spiegelt sich im Einkommensteuergesetz in der Festlegung des steuerpflichtigen Gesamteinkommens wider, das sich als Summe der Einkünfte aus den verschiedenen Einkunftsarten ergibt, ebenso in der Anerkennung des Nettoprinzips bei der Einkunftsermittlung sowie in der Berechnung des steuerpflichtigen Gewinns aus unternehmerischen Tätigkeiten durch Betriebsvermögensvergleich. Die Rechtsprechung des Bundesverfassungsgerichts lässt erkennen, dass die Leitidee der umfassenden Einkommensbesteuerung auch im Grundgesetz verankert ist.

\footnotetext{
$1 \quad$ Für die Durchsicht des Manuskripts und für kritische Anmerkungen danke ich Bodo Hilgers und Dirk Schindler (Zentrum für Finanzen und Ökonometrie, Universität Konstanz).
} 
Auch wenn sich die Einkommensteuergesetze der Industriestaaten in vielen Regelungen unterscheiden, so ist eine Orientierung an dem SHS-Standard der umfassenden Einkommensteuer auch im internationalen Vergleich erkennbar. Ein Spezifikum der umfassenden Einkommensbesteuerung ist die unbeschränkte Steuerpflicht, welche die inländischen und die ausländischen Einkünfte zu einem umfassenden Welteinkommen addiert und der nationalen Einkommensteuer unterwirft. Bilaterale Doppelbesteuerungsabkommen respektieren das nationale Recht zur Besteuerung des Welteinkommens ausdrücklich, zielen aber darauf $\mathrm{ab}$, durch geeignete Maßnahmen die diskriminierende doppelte Besteuerung von Einkünften im Quellenland (beschränkte Steuerpflicht) und im Wohnsitzland (unbeschränkte Steuerpflicht) zu vermeiden. Damit hat der SHS-Standard auch Eingang in die internationale Steuerordnung gefunden.

Im Kontrast zur allgemeinen Orientierung am SHS-Standard weist das Einkommensteuerrecht in allen Industriestaaten im Detail eine Vielzahl von Regelungen auf, die das Prinzip der umfassenden Einkommensbesteuerung aushöhlen. Die Gründe dafür sind vielfältig und reichen von der Vereinfachung der Verwaltung, dem Mangel an objektiven Kontrollen, dem Setzen von steuerlichen Lenkungsanreizen bis $\mathrm{zu}$ verteilungspolitisch motivierten Maßnahmen zur steuerlichen Entlastung.

In einigen europäischen Ländern sind jedoch in den neunziger Jahren Steuerreformschritte gesetzt worden, die eine neue Qualität der Abkehr von dem Prinzip der umfassenden Einkommensbesteuerung darstellen. Die duale Einkommensbesteuerung in den skandinavischen Ländern spaltet die Kapitaleinkünfte von den sonstigen persönlichen Einkünften $a b$ und wendet auf die Kapitaleinkommen einen niedrigeren Steuersatz an (Sørensen 1998, Cnossen 1999, Wagner 2000b). Dies bedeutet eine systematische und nicht bloß punktuelle Abweichung vom Grundprinzip der synthetischen Einkommensbesteuerung. Ähnliche Zielsetzungen liegen auch den Steuerreformen in Österreich (Genser 1996, Wagner 2000a) und in Italien (Bordignon et al. 1999) zu Grunde, wo Kapitaleinkünfte aus Zinsen und Dividenden einer Abgeltungssteuer mit einem proportionalen Steuersatz unterworfen sind, der deutlich unter dem Spitzensatz der Einkommensteuer liegt. Kritiker der dualen Einkommensbesteuerung und der Abgeltungssteuer sehen in diesen Reformschritten eine Abkehr von der umfassenden Einkommensbesteuerung und beklagen, dass damit die Steuergerechtigkeit verletzt und das Steuersystem weiter verkompliziert werden.

Ziel des vorliegenden Beitrags ist es, aus ökonomischer Sicht zu untersuchen, ob das theoretische Konzept einer umfassenden Einkommensteuer einfacher und gerechter ist als jenes einer dualen Einkommensteuer skandinavischer Prägung. Der weitere Aufbau der Arbeit ist wie folgt. Kapitel 2 kontrastiert die Vorzüge der umfassenden Einkommensteuer mit ihren konzeptionellen und administrativen Schwachstellen. Kapital 3 stellt das Konzept der dualen Einkommenssteuer vor. In Kapitel 4 werden administrative und distributive Vorteile aufgeführt, die erzielbar sind, wenn die aktuelle deutsche Einkommensteuer, die sich als praktische Umsetzung einer umfassenden Einkommensteuer versteht, von einer dualen Einkommensteuer abgelöst würde. Kapitel 5 fasst zusammen und räumt aus wohlfahrtsökonomischer Sicht der dualen Einkommensbesteuerung den Vorzug gegenüber der umfassenden Einkommensteuer ein. 
Die traditionelle Finanzwissenschaft betont die Vorzüge des SHS-Konzepts der umfassenden Einkommensteuer gegenüber historischen Alternativkonzepten wie Rohertragsteuern, Personal- und Standessteuern oder Schedulensteuern (vgl. Homburg 1997). Mit der umfassenden Einkommensteuer wird den auf Adam Smith zurückgehenden Grundsätzen (vgl. Homburg 2001, Kap. 2) der Steuergerechtigkeit, has heißt einer Steuerbemessung nach der ökonomischen Leistungsfähigkeit der Bürger, aber auch der Bestimmtheit und Einfachheit besser entsprochen als durch alternative Besteuerungsformen.

Die Besteuerung des umfassenden Einkommens ist besonders einfach, weil die Steuerbemessung für jeden Bürger nach einem einzigen Steuersatz erfolgt, der auf sein Gesamteinkommen angewendet wird. Dies vereinfacht auch die Steuereinhebung und die Steuerkontrolle, weil durch die Festlegung des synthetischen Gesamteinkommens als Steuerbasis Probleme der Abgrenzung von Einkommen aus unterschiedlichen Quellen und der korrekten Zuordnung von Einkünften irrelevant sind. Einkünfte aus der Vermietung einer Wohnung erhöhen das Gesamteinkommen und damit die Einkommensteuerbelastung in gleicher Weise, unabhängig davon, ob die vermietete Wohnung zum Betriebsvermögen oder zum Privatvermögen des Vermieters gerechnet wird.

Die Besteuerung des umfassenden Einkommens ist gerecht, weil Bürger mit gleichem synthetischen Einkommen die gleiche Steuerbasis haben und damit auch die gleiche Steuerbelastung tragen, unabhängig davon, aus welchen Einkunftsquellen ihr Gesamteinkommen gespeist wird. Damit ist die Forderung nach horizontaler Gerechtigkeit erfüllt, die das Leistungsfähigkeitsprinzip impliziert. Bürger mit gleicher ökonomischer Leistungsfähigkeit, das heißt gleichem umfassenden Einkommen, erleiden durch die Einkommensteuer eine gleich hohe Einkommenseinbuße und verfügen nach Steuern über ein gleich großes Nettoeinkommen, aus dem sie ihren Konsum finanzieren können. Demgegenüber werden ökonomisch leistungsfähigere Bürger in der Regel mit einem höheren Einkommensteuersatz belegt (vertikale Gerechtigkeit), dessen Festlegung aus der progressiven Ausgestaltung des Steuertarifs folgt.

\subsection{Konzeptionelle Schwachstellen der umfassenden Einkommensbesteuerung}

Die in einer Periodenbetrachtung intuitiv plausible Heranziehung des umfassenden Einkommens als Indikator der ökonomischen Leistungsfähigkeit und als Steuerbasis weist jedoch in einer Mehrperiodenbetrachtung eine fundamentale konzeptionelle Schwäche auf: Die Besteuerung des umfassenden Einkommens über mehrere Perioden hinweg diskriminiert systematisch Bürger, die aus ihrem Periodeneinkommen Ersparnisse zurücklegen, um daraus Konsumausgaben in späteren Lebensperioden $\mathrm{zu}$ finanzieren. Die umfassende Einkommensteuer verstößt damit gegen das Prinzip einer (periodenübergreifenden) horizontalen Gerechtigkeit, weil von zwei Bürgern mit barwertgleichen Lebenseinkommen jener eine höhere Einkommensteuerbelastung zu tragen hat, dem sein Lebenseinkommen früher zufließt und der in seiner Lebenskonsumplanung mehr Ersparnisse bilden muss.

Tabelle 1 veranschaulicht die steuerliche Belastung durch eine umfassende Einkommensteuer für unterschiedliche Lebenseinkommensprofile in einem Zweiperiodenmodell. Entlang den Spalten 2 und 3 fließt einem repräsentativen Bürger ein barwertgleiches Lebenseinkommen 
$\mathrm{zu}$, das er als Periodeneinkommen versteuern muss. Der entsprechende Lebenskonsumplan wird durch Sparen und Kreditaufnahme auf einem perfekten Kapitalmarkt finanziert. Die Steuerbelastung steigt, je größer der Anteil des Lebenseinkommens ist, das bereits in der ersten Lebensperiode zufließt. Gegenüber der tariflichen Steuerbelastung von $40 \%$ liegt nach Spalte 2 die Einkommensteuerbelastung um 7 Prozentpunkte (rund 18\%) höher, wenn das gesamte Lebenseinkommen bereits in der ersten Periode zufließt, bzw. um fast 13 Prozentpunkte (rund 30\%) niedriger, wenn das Einkommen erst in der zweiten Lebensperiode zufließt. Diese unterschiedliche Steuerbelastung von barwertgleichen Lebenseinkommen ist zweifelsohne ein Verstoß gegen das Prinzip der horizontalen Gerechtigkeit. Die Spalten 3 und 4 zeigen den gleichen qualitativen Befund bei alternativen Normierungen, wenn z.B. anstelle der proportionalen Kürzung des Periodenkonsums nur der Zweitperiodenkonsum eingeschränkt wird (Spalte 3) bzw. der Barwert des Lebenseinkommens vor Steuern so ausgeweitet wird, dass in beiden Perioden ein fixer, vorgegebener Periodenkonsum finanziert werden kann (Spalte 4).

Das Belastungsmuster durch die umfassende Einkommensteuer ändert sich, wenn die Schuldzinsen nicht die Steuerbemessungsgrundlage verringern. In einem Einkommensteuersystem ohne Schuldzinsenabzug kommt der steuerliche Entlastungseffekt durch eine Kreditfinanzierung des Lebenskonsums nicht zum Tragen und die Belastung des Lebenseinkommens stimmt mit der tariflichen Steuerbelastung überein. Die diskriminierende Mehrbelastung des Lebenseinkommens durch die Verzinsung der Ersparnisse bleibt jedoch in dem in Tabelle 1 angegebenen Ausmaß wirksam.

Tabelle 1:

Belastung des Barwertes des Lebenseinkommens durch eine umfassende Einkommensteuer bei unterschiedlichem Einkommenszufluss

\begin{tabular}{|c|c|c|c|}
\hline $\begin{array}{c}\text { Anteil des Erstperioden- } \\
\text { einkommens am Barwert } \\
\text { des Lebenseinkommens }\end{array}$ & $\begin{array}{c}\text { Lebenseinkommen fix, } \\
\text { gleicher realer } \\
\text { Periodenkonsum }\end{array}$ & $\begin{array}{c}\text { Lebenseinkommen fix, } \\
\text { Erstperiodenkonsum } \\
\text { fix }\end{array}$ & $\begin{array}{c}\text { realer Periodenkonsum } \\
\text { fix, Lebenseinkommen } \\
\text { angepasst }\end{array}$ \\
\hline $0 \%$ & $28,4 \%$ & $32,8 \%$ & $29,7 \%$ \\
\hline $10 \%$ & $30,3 \%$ & $34,2 \%$ & $31,4 \%$ \\
\hline $20 \%$ & $32,1 \%$ & $35,7 \%$ & $33,6 \%$ \\
\hline $30 \%$ & $34,0 \%$ & $37,1 \%$ & $34,7 \%$ \\
\hline $40 \%$ & $35,8 \%$ & $38,6 \%$ & $36,3 \%$ \\
\hline $50 \%$ & $37,7 \%$ & $40,0 \%$ & $37,9 \%$ \\
\hline $60 \%$ & $39,5 \%$ & $41,4 \%$ & $39,5 \%$ \\
\hline $70 \%$ & $41,4 \%$ & $42,9 \%$ & $41,4 \%$ \\
\hline $80 \%$ & $43,2 \%$ & $44,3 \%$ & $43,2 \%$ \\
\hline $90 \%$ & $45,1 \%$ & $45,8 \%$ & $45,1 \%$ \\
\hline $100 \%$ & $47,0 \%$ & $47,2 \%$ & $47,0 \%$ \\
\hline
\end{tabular}

Quelle: eigene Berechnung (Einkommensteuersatz 40\%, Zinssatz 100\%, Inflation 50\%, Nominalwertprinzip, Schuldzinsenabzug) 
Ursache des Verstoßes gegen die horizontale Gerechtigkeit ist die Besteuerung des Zinsertrages, welcher auf einem perfekten Kapitalmarkt erzielt wird und der Barwertberechnung zu Grunde liegt. Diese diskriminierende Wirkung ist eine konzeptionelle Schwäche des Prinzips der umfassenden Einkommensteuer, die auf einem periodenbezogenen, statischen Gerechtigkeitskonzept basiert, welches nicht konsistent auf ein Mehrperiodensystem übertragen werden kann (vgl. Boadway/Bruce 1984).

Eine zweite konzeptionelle Schwäche der umfassenden Einkommensbesteuerung ist die systematische Diskriminierung von Sachkapitalakkumulation gegenüber Humankapitalakkumulation. Nach dem SHS Standard wird im umfassenden Einkommen nur der Zuwachs an Real- und Finanzkapital in einer Steuerperiode berücksichtigt, der Zuwachs an Humankapital hingegen nicht.

Für den steuerpflichtigen Bürger bedeutet dies einerseits, dass nicht nur die laufenden Erträge aus seinem Sachkapitalvermögen (Zinsen, Dividenden, Gewinnausschüttungen) sondern auch Wertsteigerungen seines Sachkapitals (Wertpapiere, Grundstücke, Firmenwerte) steuerpflichtiges Einkommen darstellen. Damit wird dem Prinzip der horizontalen Gerechtigkeit entsprochen, weil grundsätzlich jeder Kapitalgewinn durch Veräußerung des Kapitalgutes realisiert werden kann und aus Sicht der steuerlichen Leistungsfähigkeit eine Gleichbehandlung von laufenden Kapitalerträgen und Kapitalgewinnen geboten ist. Demgegenüber werden Einkünfte aus Humankapital nach dem SHS-Standard nur im Ausmaß der laufenden Erträge als steuerpflichtiges Einkommen erfasst (Arbeitslohn, Unternehmerlohn), während Wertsteigerungen im Humankapital das umfassende Einkommen nicht erhöhen. Aus steuertechnischer Sicht ist dies verständlich, denn für das Gut Humankapital gibt es im Gegensatz zu Sachkapitalgütern keinen Markt, zumindest nicht mehr seit der Abschaffung der Sklaverei. Nach dem SHS-Standard sind Kapitaleinkommen grundsätzlich durch Vermögensvergleich zu ermitteln, während Arbeitseinkommen als Nettoüberschuss der Arbeitslöhne über die Werbungskosten $\mathrm{zu}$ berechnen sind. In der praktischen Umsetzung der umfassenden Einkommensbesteuerung wird dieser asymmetrischen Behandlung von Sach- und Humankapital explizit Rechnung getragen durch den vielfach kritisierten Dualismus in der Ermittlung der Einkünfte, der sich auch im deutschen Einkommensteuergesetz niedergeschlagen hat (vgl. Wenger 1983, Wagner 2000b). Für die Berechnung des steuerpflichtigen Gewinns aus unternehmerischer Tätigkeit ist der Betriebsvermögensvergleich vorgeschrieben, für die Ermittlung der Einkünfte aus unselbständiger Arbeit die Überschussrechnung. Dass nach dem deutschen Einkommensteuergesetz auch Kapitaleinkünfte im privaten Bereich (Vermietung, Kapitalanlagen) durch Überschussrechnung und nicht durch Vermögensvergleich zu ermitteln sind, ist eine bewusste Abweichung vom SHS-Prinzip.

Tabelle 2 veranschaulicht die diskriminierende Steuerbelastung der umfassenden Einkommensteuer bei ertragsgleichen Investitionen in Sach- bzw. Humankapital in einem Zweiperiodenmodell. Gegenüber einer ausschließlichen Humankapitalinvestition, bei der die Gesamtsteuerbelastung mit dem tariflichen Einkommensteuersatz übereinstimmt, steigt die Gesamtsteuerbelastung, je größer der Anteil der Ersparnis ist, die in Sachkapital angelegt wird. Die Steuerbelastung sinkt unter die tarifliche Belastung, wenn ein Teil der Humankapitalinvestition über Kreditaufnahme finanziert wird und die Schuldzinsen abzugsfähig sind (Tabelle 2, letzte Zeile). 
Tabelle 2

Belastung des Barwertes des Lebenseinkommens durch eine umfassende Einkommensteuer bei unterschiedlicher Investition in Sachkapital und Humankapital

\begin{tabular}{|c|c|}
\hline $\begin{array}{c}\text { Anteil der } \\
\text { Humankapitalinvestition } \\
\text { an der Gesamtinvestition }\end{array}$ & $\begin{array}{c}\text { Lebenseinkommen fix, } \\
\text { gleicher realer } \\
\text { Periodenkonsum }\end{array}$ \\
\hline $0 \%$ & $48,6 \%$ \\
\hline $10 \%$ & $47,9 \%$ \\
\hline $20 \%$ & $47,3 \%$ \\
\hline $30 \%$ & $46,6 \%$ \\
\hline $40 \%$ & $45,8 \%$ \\
\hline $50 \%$ & $45,0 \%$ \\
\hline $60 \%$ & $44,1 \%$ \\
\hline $70 \%$ & $43,2 \%$ \\
\hline $80 \%$ & $42,2 \%$ \\
\hline $90 \%$ & $41,2 \%$ \\
\hline $100 \%$ & $40,0 \%$ \\
\hline $110 \%$ & $38,8 \%$ \\
\hline
\end{tabular}

Quelle: eigene Berechnung (Einkommensteuersatz 40\%, Zinssatz = Ertragsrate der Humankapitalinvestition $=100 \%$, Inflation 50\%, Nominalwertprinzip, Schuldzinsenabzug)

Ursache des Verstoßes gegen die horizontale Gerechtigkeit ist die ungleiche Behandlung von Humankapital- und Sachkapitalzuwächsen in der Berechnung des umfassenden Einkommens. Horizontale Gerechtigkeit würde im konkreten Beispielsfall erreichbar sein, wenn entweder der Zuwachs an Humankapital ebenfalls das umfassende Einkommen erhöht oder wenn der Zuwachs an Sachkapital aus dem umfassenden Einkommen ausgeschieden wird (vgl. Kaplow 1996). Die Besteuerungsniveaus würden in den beiden Varianten allerdings unterschiedlich sein (im gewählten Beispiel 48,2\% bei Besteuerung des gesamten Kapitalzuwachses bzw. $40 \%$ bei Besteuerung der Cashflows).

\subsection{Schwächen in der steuertechnischen Umsetzung der umfassenden Einkommensteuer}

Die Ermittlung des umfassenden Einkommens erfordert die Kenntnis der Vermögenswerte zu den Bewertungsstichtagen. Der korrekte Marktpreis eines Kapitalgutes lässt sich allerdings erst im Veräußerungsfall beobachten. So lange Kapitalgüter im Vermögen gehalten und nicht verkauft werden, muss man mit Näherungswerten operieren, die lediglich eine Schätzgröße für den tatsächlichen Vermögenszuwachs liefern. Selbst wenn der Kapitaleigentümer den Marktwert seiner Anlagegüter genau kennt, hat er einen Anreiz, gegenüber der Finanzverwaltung den Vermögenszuwachs $\mathrm{zu}$ untertreiben und durch eine zeitliche Verschiebung der Einkommensbesteuerung einen Zinsgewinn zu erzielen, der den Barwert der Steuerbelastung verringert. In der Besteuerungspraxis offenbart sich dieses strategische Verhalten durch die Bildung von stillen Reserven, die Dotierung von Rückstellungen und durch Wertberichtigungen, die in Deutschland sogar durch das Niedrigstwertprinzip des Handelsrechts gesetzlich normiert sind. Gegenüber dem SHS-Standard wird damit der Vermögenszuwachs 
in den Steuerbilanzen systematisch unterschätzt und das Prinzip der umfassenden Einkommensbesteuerung ausgehöhlt.

Eine weitere systematische Aushöhlung des SHS-Standards tritt ein, wenn das Einkommensteuergesetz an Stelle der unmittelbaren Besteuerung des Vermögenszuwachses die Besteuerung auf den Zeitpunkt der Realisation verschiebt (Realisationsprinzip). Dies gilt für alle Vermögenszuwächse im privaten Bereich, wo Kapitalgewinne erst steuerpflichtig werden, wenn beim Verkauf vor Ablauf einer im Einkommensteuergesetz normierten Behaltefrist ein Veräußerungsgewinn erzielt wird. In Deutschland wird die Bedeutung der Kapitalgewinne künftig weiter ansteigen, weil die Unternehmensteuerreform 2000 durch die Senkung des Körperschaftsteuersatzes auf einbehaltene Gewinne auf $25 \%$ einen Anreiz zur Thesaurierung von Gewinnen in Kapitalgesellschaften setzt. Das Realisationsprinzip gilt aber auch für Vermögenszuwächse im Rahmen der Altersvorsorge von unselbständig Beschäftigten, wo der individuelle Vermögensaufbau durch Rentenversicherungsbeiträge, durch Pensionsansprüche im öffentlichen Dienst oder durch zugesagte Firmenpensionen nicht in die Einkommensteuerbemessung eingeht.

Um den Freiraum in der Vermögensbewertung zu verringern und die Steuerprüfung zu vereinfachen, sieht das Einkommensteuergesetz das Nominalwertprinzip vor. Dies bedeutet, dass Abschreibungsbeträge nach den historischen Anschaffungs- oder Herstellungskosten bemessen werden oder dass Verluste aus früheren Veranlagungsperioden vorgetragen werden. Bei Inflation verzerrt die Verwendung von nominellen Geldgrößen früherer Veranlagungsjahre die Vermögensbewertung und verletzt damit auch die horizontale Gerechtigkeit.

Schließlich normiert das deutsche Einkommensteuergesetz eine Vielzahl spezieller Ausnahmen vom Grundsatz der umfassenden Einkommensbesteuerung, indem bestimmte Einkünfte ausdrücklich von der Einkommensteuer ausgenommen sind. Für die Aushöhlung des SHS-Standards, z.B. durch steuerfreie Veräußerungserlöse im privaten Bereich (nach Ablauf der Behaltefrist), durch einkommensteuerfreie Vermögenszuwächse nach Erbschaften und Schenkungen, durch steuerfreie Kapitalerträge (Lebensversicherungen, Zinsen und Dividenden innerhalb des Sparerfreibetrags), durch steuerfreie Lohneinkommen (Lohnzuschläge, fringe benefits), etc., werden unterschiedliche steuerpolitische Motive genannt. In ihrer Gesamtheit verletzen aber alle diese unsystematischen, unübersichtlichen und verwaltungsaufwendigen Einzelregelungen zur Vereinfachung der Verwaltung, zur Entlastung einkommensschwacher Haushalte oder zur meritorischen Verhaltenssteuerung sowohl die horizontale als auch die vertikale Gerechtigkeit und damit die generelle Gerechtigkeitsnorm des Leistungsfähigkeitsprinzips, auf welche sich die umfassende Einkommensbesteuerung stützt.

\section{Das Grundkonzept der dualen Einkommensteuer der nordischen Staaten}

Die Einkommensteuerreformen der Industrieländer verfolgen seit Mitte der achtziger Jahre das Ziel, die Steuersätze zu senken, um die Wettbewerbschancen im globalen Standortwettbewerb zu verbessern und gleichzeitig die Steuerbemessungsgrundlagen $\mathrm{zu}$ verbreitern, um Steuerschlupflöcher zu schließen und das Steueraufkommen stabil zu halten, damit die Konsolidierung der öffentlichen Haushalte nicht gefährdet wird. 
Im Zuge dieser Reformbestrebungen nach dem ,tax cut cum base broadening“ Vorbild der USA haben insbesondere die skandinavischen Staaten neue Wege in der Einkommensbesteuerung beschritten. Ziel der nordischen Einkommensteuerreformen war einerseits der Abbau der Verzerrungswirkungen der traditionellen Kapitaleinkommensbesteuerung (King/Fullerton 1984) und andererseits eine Anpassung der Steuersysteme an die gestiegene internationale Kapitalmobilität (Sørensen 1998, ix). Der Reformbedarf war in den skandinavischen Staaten besonders hoch, weil die im internationalen Vergleich hohen tariflichen Sätze internationale Investoren abschreckten, gleichzeitig aber für inländische Investoren einen Anreiz boten, Steuerausweichstrategien $\mathrm{zu}$ entwickeln, die dazu geführt haben, dass die effektive Steuerbelastung von Kapitaleinkünften weit geringer war als die nominellen Sätze.

Die Steuerreformen der skandinavischen Staaten (Tabelle 3) markieren eine Abkehr von der synthetischen Einkommensteuer hin zu einer analytischen Einkommensteuer, welche die Erträge aus Sachkapital (Finanzkapital und Realkapital) niedriger besteuert als die Erträge aus Humankapital (Löhne und Gehälter, Unternehmerlohn). Die zwei unterschiedlichen Tarife für Kapital- und Arbeitseinkünfte haben den skandinavischen Einkommensteuersystemen den Namen ,duale Einkommensteuer“ gegeben. Tabelle 3 charakterisiert die Reformschritte von der synthetischen zur dualen Einkommensteuer in vier skandinavischen Ländern. Dabei zeigt sich, dass Dänemark, der Vorreiter in der Einführung einer dualen Einkommensteuer, sich seit 1994 wieder eher einer synthetischen Einkommensbesteuerung zugewendet hat. Finnland, Norwegen und Schweden haben hingegen eine duale Einkommensteuer eingeführt, die Kapitaleinkünfte mit einem Proportionalsatz belegt, während Arbeitseinkünfte nach einem progressiven Tarif besteuert werden. Der proportionale Kapitalsteuersatz ist dabei kleiner oder gleich dem niedrigsten Grenzsteuersatz für Arbeitseinkünfte.

Eine Besonderheit der dualen Einkommensteuer ist die Anwendung des progressiven Steuertarifs nicht nur auf Einkünfte aus unselbständiger Arbeit, sondern auch auf die Arbeitseinkünfte von Unternehmern, den Unternehmerlohn, und auf den reinen Profit. Diese beiden Gewinnformen sind ebenso wie die Verzinsung des Eigenkapitals Bestandteile des Unternehmensgewinns. Unternehmerlohn und Eigenkapitalverzinsung werden als kalkulatorische Faktorerträge in den Einkommensteuerbilanzen nicht separat ausgewiesen, weil der gesamte Unternehmensgewinn mit dem gleichen Steuersatz belegt wird. Die duale Einkommensteuer bietet jedoch einen Anreiz, den Unternehmerlohn gering auszuweisen, um der höheren Arbeitsbesteuerung zu entgehen. Daher sind objektiv nachprüfbare, gesetzliche Regelungen zur Bestimmung des Unternehmerlohns und des reinen Profits notwendig. Diese Aufspaltung des Unternehmensgewinns wird in den drei Ländern in der Form vorgenommen, dass in Einzelunternehmen und Personengesellschaften die marktübliche Eigenkapitalverzinsung als kalkulatorische Kostengröße ermittelt wird. Die Differenz zum unternehmerischen Gesamtgewinn ist die Summe aus Unternehmerlohn und reinem Profit, die mit dem progressiven Steuertarif für Arbeitseinkommen belastet wird.

Gerade in dieser unterschiedlichen Besteuerung der Eigenkapitalrendite und der sonstigen Gewinnkomponenten nach getrennten Tarifen unterscheidet sich die duale Einkommensteuer von Einkommensteuerreformen in anderen Industriestaaten, die gleichfalls eine niedrigere Besteuerung bestimmter Kapitaleinkünfte vorsehen (Wagner 2000). Dazu zählen insbesondere die Abgeltungssteuern auf Zinsen und Dividenden in Belgien, Österreich (Genser 1996, 1999) und Italien (Bordignon et al. 1998) oder das Halbeinkünfteverfahren in 
Deutschland (Wagner 2000). Denn Dividenden und Gewinnanteile unterliegen zwar ermäßigten Einkommensteuersätzen, tragen aber auch die Körperschaftsteuer und somit eine Doppelbelastung, durch welche die Gesamtsteuerbelastung der Eigenkapitalverzinsung auf das Niveau des höchsten Grenzsteuersatzes des progressiven Einkommensteuertarifs angehoben wird. Die Ermäßigungswirkung der Abgeltungssteuer bleibt damit auf Zinseinkünfte beschränkt.

Tabelle 3

Marginale Einkommensteuersätze in den Nordischen Staaten (einschließlich kommunaler Einkommensteuer, ohne Sozialversicherungsbeiträge)

\begin{tabular}{|c|c|c|c|}
\hline & $\begin{array}{l}\text { persönliches } \\
\text { Einkommen }\end{array}$ & $\begin{array}{c}\text { Kapital } \\
\text { einkommen }\end{array}$ & $\begin{array}{c}\text { Körperschaft- } \\
\text { steuer }\end{array}$ \\
\hline \multicolumn{4}{|l|}{ Dänemark } \\
\hline umfassende ESt (bis 1986) & $48 \%-73 \%$ & $48 \%-73 \%$ & $40 \%$ \\
\hline duale ESt (1987-1993) & $50 \%-68 \%$ & $50 \%-56 \%$ & $50 \%$ \\
\hline duale ESt (ab 1994) & $38 \%-58 \%$ & $\begin{array}{c}38 \%-44 \% \\
\text { (unter } 20.000 \mathrm{DKr} \text { ) } \\
\text { bzw. } 58 \%\end{array}$ & $34 \%$ \\
\hline \multicolumn{4}{|l|}{ Finnland } \\
\hline umfassende ESt (bis 1992) & $25 \%-56 \%$ & $25 \%-56 \%$ & $37 \%$ \\
\hline duale ESt (ab 1993) & $25 \%-56 \%$ & $25 \%$ & $25 \%$ \\
\hline \multicolumn{4}{|l|}{ Norwegen } \\
\hline umfassende ESt (bis 1991) & $26,5 \%-50 \%$ & $26,5 \%-40,5 \%$ & $50,8 \%$ \\
\hline duale ESt (ab 1992) & $28 \%-41,7 \%$ & $28 \%$ & $28 \%$ \\
\hline \multicolumn{4}{|l|}{ Schweden } \\
\hline umfassende ESt (bis 1990) & $36 \%-72 \%$ & $36 \%-72 \%$ & $52 \%$ \\
\hline duale ESt (ab 1991) & $31 \%-51 \%$ & $30 \%$ & $30 \%$ \\
\hline
\end{tabular}

Quelle: Sørensen 1998, Tab. 1.1

\section{$4 \quad$ Vorzüge einer dualen Einkommensteuer}

Obwohl eine duale Einkommensteuer durch die Anwendung von zwei Steuertarifen auf separiert auszuweisende Bemessungsgrundlagen verwaltungsaufwendiger und durch die steuerliche Entlastung der Kapitaleinkünfte nicht gerecht gestaltet erscheint, lässt sich zeigen, dass diese Einschätzung vordergründig ist und aus ökonomischer Sicht nicht aufrecht erhalten werden kann. 


\subsection{Vereinfachung der Einkommensbesteuerung}

Eine duale Einkommensteuer, die Kapitalerträge mit einer proportionalen Einkommensteuer belegt, kann als Quellensteuer bereits im Ausmaß der endgültigen Steuerbelastung eingehoben werden. Damit ist eine Verwaltungsvereinfachung verbunden, weil eine Veranlagung dieser Kapitaleinkünfte nicht mehr erforderlich ist. Sind für einen Arbeitnehmer die Einkünfte aus unselbständiger Arbeit bereits durch die Lohnsteuerzahlung des Arbeitgebers mit der endgültigen tariflichen Jahressteuer belastet, dann kann dessen Veranlagung unter einer dualen Einkommensteuer sogar gänzlich entfallen. Von den rund 25 Mio. Steuerpflichtigen mit Einkünften aus unselbständiger Arbeit in Deutschland haben nach der Lohnund Einkommensteuerstatistik 1995 etwa 800.000 auch Einkünfte aus Kapitalvermögen bezogen, die zu veranlagen waren (Statistisches Bundesamt 1999, Tab. 2.2.1). Das aktuelle Verwaltungsentlastungspotenzial beträgt jedoch ein Vielfaches dieser Zahl, weil die Veranlagungsfälle durch die Halbierung des Sparerfreibetrags auf DM 3000 ab 2000 deutlich ansteigen werden.

Vereinfacht wird aber auch die Veranlagung von Steuerpflichtigen mit gewerblichen und selbständigen Einkünften, weil die Dokumentation der Kapitaleinkünfte und der darauf geleisteten Steuervorauszahlungen entfallen kann.

Schließlich kann auf die Einhebung einer separaten Quellensteuer auf Dividenden und Gewinnanteile verzichtet werden, wenn der Körperschaftsteuersatz mit dem Proportionalsatz auf Kapitaleinkünfte zusammenfällt und daher die Gewinnausschüttungen bereits durch die Körperschaftsteuer endbesteuert sind. Diese Form der finalen Besteuerung über die Körperschaftsteuer wird bereits in Finnland, Norwegen und Schweden praktiziert (Tabelle 3).

Eine duale Einkommensteuer mit proportionaler Endbesteuerung würde auch dem deutschen Bankensektor eine erhebliche Verwaltungsentlastung bringen, weil sie den Sparerfreibetrag und die Überwachung der Freistellungsaufträge entbehrlich macht. Durch die einheitliche Quellenbesteuerung aller Kapitalerträge benötigt man keine Bagatellegrenze für Kleinsparer und die steuerliche Entlastung der Kapitalerträge erfolgt direkt durch einen niedrigeren Steuersatz anstelle einer Kürzung der Steuerbemessungsgrundlage durch den Sparerfreibetrag. Der Übergang von der Sparerfreibetragslösung zu einer dualen Einkommensteuer sollte sich im Bankensektor in geringeren Bankgebühren niederschlagen und damit vor allem Kleinanleger relativ stärker entlasten. Bei der Steuerverwaltung fallen ebenfalls Kosteneinsparungen an, die bei der Bemessung des Quellensteuersatzes berücksichtigt werden sollten. Will man Kleinsparer von der Quellensteuer auf Zinseinkommen entlasten, so lässt sich ein entsprechender allgemeiner Steuerkredit für Kleinsparer auch in den Einkommensteuertarif einbauen.

\subsection{Gerechtere Einkommensbesteuerung}

Die duale Einkommensbesteuerung bietet eine Möglichkeit, die im SHS Standard verankerte diskriminierende Doppelbesteuerung der Ersparnisse zu mildern. Zinseinkommen werden zwar nicht steuerfrei gestellt, aber die Verletzung der horizontalen Gerechtigkeit fällt bei niedrigeren Kapitalsteuersätzen weniger ins Gewicht. Darüber hinaus werden auch die negativen Auswirkungen des Nominalwertprinzips bei der Zinsenbesteuerung gemildert, wo die Inflationsabgeltung durch die Nominalverzinsung bei Einkommensteuersätzen von 50\% 
schon bei niedrigen Inflationsraten zu negativen Realzinsen nach Steuern und damit zu einem realen Wertverlust des eingesetzten Anlagekapitals führt.

Durch die Quellenbesteuerung von Kapitalerträgen können auch bisher unbesteuerte Anlagen ohne hohe Verwaltungs- und Kontrollkosten korrekt endbesteuert werden und bestehende Steuerlücken geschlossen werden. Dies gilt beispielsweise für Erträge aus Lebensversicherungen im Erlebensfall. Die Quellensteuer auf Gewinnbeteiligungen und Bonusse kann direkt vom Versicherungsunternehmen abgeführt werden, ohne dass beim Begünstigten eine nachfolgende Veranlagung erforderlich ist oder Liquiditätsengpässe auftreten.

Die duale Einkommensteuer schafft zwar nicht eine steuerliche Gleichbehandlung von verschiedenen Formen von Kapitalanlagen im persönlichen Bereich, weil die Sonderregelungen für die gesetzliche Rentenversicherung, für die Beamtenpensionen oder für Betriebspensionen weitergelten werden und Kapitalerträge aus diesen Sozialprogrammen nicht in die duale Einkommensteuer eingebunden werden. Die duale Einkommensteuer ebnet aber das Spielfeld für weitere Formen der ergänzenden, kapitalmarktbasierten Altersvorsorge, die ohne steuerliche Diskriminierung größere Akzeptanz finden und die Substitution von sinkenden Einkommensersatzraten in der gesetzlichen Alterssicherung erleichtern sollte (vgl. Donges 2000, 34).

Schließlich eröffnet die duale Tarifstruktur die Option, auf geänderte ökonomische Rahmenbedingungen (Sparverhalten, Inflation, Globalisierung) durch eine Anpassung des Proportionalsatzes auf Kapitalerträge reagieren zu können, ohne die Tarifstruktur für Arbeitseinkünfte mit zu verändern. Dieser zusätzliche Freiheitsgrad in der dualen Einkommensbesteuerung erlaubt eine parametrische Variation der Quellenbesteuerung auf Kapitalerträge. Damit lässt sich sowohl eine umfassende Einkommensteuer realisieren, wenn die Quellensteuer in Höhe des maximalen Grenzsteuersatzes auf Arbeitseinkommen festgelegt wird und im Veranlagungsweg auf die persönliche Einkommensteuerschuld angerechnet wird, als auch eine zinsbereinigte Einkommensteuer, wenn die Quellensteuer als finale Einkommensteuer festgelegt wird und der Steuersatz auf null gesenkt wird.

\section{$5 \quad$ Fazit}

Die finanzwissenschaftlich fundierte Kritik an einer Einkommensbesteuerung nach dem SHS-Standard setzt sowohl an konzeptionellen Schwachstellen als auch an den Problemen der steuertechnischen Umsetzung der umfassenden Einkommensteuer an. Eine duale Einkommensteuer vermag administrative und distributive Mängel einer umfassenden Einkommensteuer $\mathrm{zu}$ mildern, wenn auch nicht $\mathrm{zu}$ beseitigen. Die Steuerreformen der neunziger Jahre in den skandinavischen Ländern zeigen, dass eine duale Einkommensbesteuerung nicht nur theoretisch konzipiert werden kann, sondern auch im demokratischen Entscheidungsprozess als steuerpolitische Alternative mehrheitsfähig ist.

Ein weiteres ökonomisches Argument zu Gunsten einer näheren Befassung mit Spielarten einer dualen Einkommensbesteuerung ist ihre Überlegenheit aus wohlfahrtsökonomischer Sicht. Da die Einkommensteuer stets mit Effizienzeinbußen verbunden ist, muss ihre optimale Ausgestaltung in einem „Second-best"-Modellrahmen analysiert und begründet werden. Im Rahmen eines solchen Optimalsteuermodells wird ceteris paribus ein duales Einkommensteuersystem gegenüber einer umfassenden Einkommensteuer stets ein zusätz- 
liches steuerpolitisches Instrument bereitstellen und damit potentielle Wohlfahrtsgewinne gegenüber einer Besteuerung nach dem SHS Standard eröffnen. Im theoretischen Modell wird die umfassende Einkommensteuer daher bestenfalls gleichwertig, im Regelfall jedoch unterlegen sein. Kontrastierend $\mathrm{zu}$ dieser Modellsicht ist allerdings empirisch $\mathrm{zu}$ prüfen, inwieweit die demokratische Kontrolle ausreicht, um eigennützige Steuerpolitiker daran zu hindern, den zusätzlichen steuerpolitischen Gestaltungsspielraum zu nutzen, um ihrer Wählerklientel Vorteile zuzuschanzen und nicht das Gesamtwohl der Gesellschaft im Auge zu haben. Aber dieses Problem ist in der Steuerpolitik auf Grund der vielfältigen Möglichkeiten Steuerbegünstigungen einzuräumen allgegenwärtig und kann nicht als ein Argument gegen das Konzept einer dualen Einkommensteuer vorgebracht werden.

\section{Literatur}

Boadway, Robin/Bruce, Neil. A general proposition on the design of a neutral business tax. Journal of Public Economics 24 (1984), 231-239.

Bordignon, Massimo/Giannini, Silvia/Panthegini, Paolo. Corporate taxation in Italy: An analysis of the 1998 reform. Finanzarchiv 56 (1999), 335-362.

Cnossen, Sijbren. Taxing capital income in the northern countries : A model for the European Union? Finanzarchiv 56 (1999), 18-50.

Donges, Jürgen et al. Abgeltungssteuer bei Kapitaleinkommen. Schriftenreihe des Frankfurter Instituts, Bd. 37, Bad Homburg 2000.

Genser, Bernd. Austria's steps towards a dual income tax. L.Muten et al. (eds): Towards a dual income tax? Kluwer, Rotterdam 1996, 69-89.

Genser, Bernd. Konsumorientierung - Realisierungschancen in Österreich. Ch. Smekal/R. Sendlhofer/H. Winner (Hrsg.): Einkommen versus Konsum. Ansatzpunkte zur Steuerreformdiskussion. Physica-Verlag, Heidelberg 1999, 197-215.

Homburg, Stefan. Soll die klassische Einkommensteuer wiederbelebt werden? M.Rose (Hrsg.): Standpunkte zur aktuellen Steuerreform. Recht und Wirtschaft, Heidelberg 1997, 107-114.

Homburg, Stefan. Allgemeine Steuerlehre. 2.Aufl., Vahlen, München 2001.

Kaplow, Louis. On the divergence between ideal and conventional income-tax treatment of human capital. American Economic Review 86 (1996), 347-352.

King, Mervyn/Fullerton, Don. The taxation of income from capital. Chicago UP, 1984.

Sфrensen, Peter. Fro $m$ the global income tax to the dual income tax: Recent tax reforms in the northern countries. International Tax and Public Finance 1 (1994), 57-79.

Sørensen, Peter (ed.). Tax policy in the nordic countries. Macmillan, London et al. 1998.

Wagner, Franz. Zinsbesteuerung in Form einer definitiven Abgeltungssteuer. H. Mayer (Hrsg.): Unternehmensbesteuerung - Perspektiven der Besteuerung. Boorberg, Stuttgart et al. 2000a, 81-96.

Wagner, Franz. Korrektur des Einkünftedualismus durch Tarifdualismus - Zum

Konstruktionsprinzip der Dual Income Taxation. Steuer und Wirtschaft 4/2000b, 431438.

Wagner, Franz. Deutschland bei Österreich in der Steuerlehre: Die falsche Lektion gelernt. U.Wagner (Hrsg.): Zum Erkenntnisstand der Betriebswirtschaftslehre am Beginn des 21. Jahrhunderts. Duncker \& Humblot, Berlin 2001, 431-449.

Wenger, Ekkehard. Gleichmäßigkeit der Besteuerung von Arbeits- und Vermögenseinkünften. Finanzarchiv 44 (1983), 207-252. 